\title{
SOM-BASED K-NEAREST NEIGHBORS SEARCH IN LARGE IMAGE DATABASES
}

\author{
Zaher AGHBARI \\ Graduate School of Information Science and Elec. Eng., Kyushu University, Japan \\ zaher@is.kyushu-u.ac.jp
}

Kun-Seok OH

College of Engineering, Chosun University, Korea

okseok38@hotmail.com

\section{Yaokai FENG}

Graduate School of Information Science and Elec. Eng., Kyushu University, Japan fengyk@db.is.kyushu-u.ac.jp

\section{akifumi MAKINOUCHI}

Graduate School of Information Science and Elec. Eng., Kyushu University, Japan akifumi@is.kyushu-u.ac.jp

\begin{abstract}
We address the problem of $K$-Nearest Neighbors (KNN) search in large image databases. Our approach is to cluster the database of $n$ points (i.e. images) using a self-organizing map algorithm. Then, we map each cluster into a point in onedimensional distance space. From these mapped points, we construct a simple, compact and yet fast index structure, called array-index. Unlike most indexes of $\mathrm{KNN}$ algorithms that require storage space exponential in dimensions, the arrayindex requires a storage space that is linear in the number of generated clusters. Due to the simplicity and compactness of the array-index, the experiments show that our method outperforms other well know methods.
\end{abstract}

\section{INTRODUCTION}

A wide range of database applications requires the ability to answer queries based on similarity. In a database of $n$ points, given a query point $\mathbf{q}$ (a $d$ dimensional vector), we want to return the $K$ points in the database that are 
most similar to q. Those $K$ points are called the $K$-Nearest Neighbors (KNN) to q. Finding KNN points is one of the most expensive operations in highdimensional databases, such as image databases [1], time series databases [2], data mining [3], etc. However, KNN search is an essential requirement to most of these applications.

In this paper, we propose a new KNN search approach based on clustering the image data and then using the representative vectors of these clusters to build a simple, compact and yet efficient index structure. First, using a wavelet transform method, we extract the color feature of each image in the database; thus, mapping an image into a point in a high-dimensional feature space. Then, these high-dimensional points are clustered based on their similarity using a self-organizing map (SOM) algorithm. Each formed cluster $C_{i}$ is represented by a $d$-dimensional vector $\mathbf{m}_{i}$, called codebook vector (CBV), where $d$ is the dimensionality of the input points.

From among the CBVs, a reference point $\mathbf{R}$ is selected, then the distance $D\left(\mathbf{m}_{i}, \mathbf{R}\right)$ between each $\mathbf{m}_{i}$ and $\mathbf{R}$ is computed. Using $\mathbf{R}$ and the computed distances, we map the CBVs of the clusters into a 1-dimensional distance space in which the mapped clusters are ordered based on their similarity to $\mathbf{R}$. Since the number of clusters $N_{C}$ is very small as compared to the number of images $N_{I}$ in the database, we can use a simple sorted array structure, called arrayindex, to index these clusters. Using a simple sorted array structure allows us to find the most similar $C_{i}$, called a winner node (WN), to a given $\mathrm{q}$ using the fast binary search algorithm whose complexity is $O\left(\log N_{C}\right)$. As a result, fast $\mathrm{KNN}$ search in large image databases is achieved.

The rest of this paper, is organized as follows: Section 2 describes some of the related work. Section 3 discusses how to generate and cluster the data, then build the array-index. In Section 4, we present the KNN search algorithm. The experiments are discussed in Section 5. Finally, we conclude the paper in Section 6.

\section{RELATED WORK}

The problem of finding the exact, or approximate, $\mathrm{KNN}$ points in a database to a given query point has been addressed by a large number of works. Most of these works have provided good solutions for the low-dimensionality case, but for the high-dimensionality case there have been very little progress. The following are some examples of the related work.

Multi-dimensional index structures, such as the $R^{*}$-tree [4] is known to perform well as compared to many other multi-dimensional index structures. However, performance of the $\mathrm{R}^{*}$-tree degrades as dimensionality increases [9]. Newly proposed trees such as the X-tree [5] improves performance over that of the $\mathrm{R}^{*}$-tree by extending the overflown nodes into super nodes. Another new 
index, called the A-tree [10], improves performance in high-dimensional space by storing virtual bounding rectangles instead of the actual MBRs.

Other methods apply "dimensionality reduction" techniques on the data points before inserting them into the trees. The QBIC system [11] of IBM uses a bounding method to map the high-dimensional color histograms of images into points in a 3-dimensional space and then organizes those points by an $\mathrm{R}^{*}$-tree. Chakrabarti et. al. [12] utilizes the Principle Component Analysis method to reduce the dimensions of the locally correlated clusters, then each locally correlated cluster is indexed by a hybrid tree. The resulting hybrid trees are then joined by a global index. However, this system requires expensive preprocessing of the data to find the correlated clusters and reduce the dimensions.

Some other algorithms are proposed to return exact KNN answer points, others return approximate $\mathrm{KNN}$ answer points. Roussopoulos et. al. [14] proposed an algorithm that starts at the root of the tree and performs a depth-first traversal to find the exact KNN points. Berchtold et. al [7] proposed a different approach that precomputes the result of any nearest neighbor search that corresponds to a computation of a voronoi cell of each data point. Then, they use an X-tree to store the voronoi cells. Other approaches, such as the works proposed by Kleinberg [8] and by Brin [15], have settled for approximate KNN in return of better performance. Although finding approximate $\mathrm{KNN}$ answer points to a given query point is of use in some applications, finding exact KNN answer points is our goal in this paper.

\section{BUILDING THE INDEX}

One of the goals of this paper is to build a simple, compact and yet fast index structure for our image database. Hence, the core of our new approach is to reduce the size of the index structure in order to speed up the KNN search. The size-reduction of the index structure is achieved by, first, clustering the data points (i.e. images) and, then, indexing the clusters rather than the data points themselves, as explained below.

\subsection{Wavelet Transformation}

We are using a Haar wavelet transform to decompose images into several frequency bands and then compute a feature vector from these bands. As a result of using the Haar wavelet transform, the generated feature vectors are compact since we can use only a few coefficients to sufficiently represent the image color content. Moreover, the Haar wavelet basis is the simplest wavelet basis, in terms of implementation, and the fastest to compute [16]. 


\subsection{SOM-Based Clustering}

According to [17], the SOM is unsupervised neural network that maps highdimensional input data $\Re^{n}$ onto a usually two-dimensional output space while preserving the topological relations (similarities) between the data items. The SOM consists of nodes (neurons) arranged in a two-dimensional rectangular or hexagonal grid. A codebook vector $(\mathrm{CBV}) \mathbf{m}_{\mathbf{i}} \in \Re^{n}$ is associated with every node $i$. An input vector $\mathbf{x} \in \Re^{n}$ is compared with the $\mathbf{m}_{\mathbf{i}}$, and the bestmatching-node (BMN), which has the smallest Euclidean distance $D\left(\mathbf{x}, \mathbf{m}_{\mathbf{i}}\right)$, see Equation 1, is selected. The input is thus mapped onto the location of the selected BMN.

$$
D\left(\mathbf{x}, \mathbf{m}_{\mathbf{i}}\right)=\left(\sum^{N_{d}}\left(\mathbf{x}-\mathbf{m}_{\mathbf{i}}\right)^{2}\right)^{1 / 2}
$$

Where, $N_{d}$ is the dimensionality of the input vectors. The CBV $\mathbf{m}_{c}$ of the BMN is adopted to match the input vector. That is done by moving $\mathbf{m}_{c}$ towards $\mathbf{x}$ by a certain fraction of the distance $D\left(\mathbf{x}, \mathbf{m}_{\mathbf{c}}\right)$. Moreover, the CBVs of nodes in the neighborhood of the BMN are moved towards $\mathbf{x}$, but to a lesser extent than the CBV of the BMN. This learning process finally leads to a topologically-ordered mapping of the input vectors. That means the similarity between data points and between clusters are represented in the map. The CBV of a node (cluster) is quite representative of all points within the node. In this paper, we will use the terms node and cluster alternatively.

\subsection{Cluster Mapping}

After generating the clusters by a SOM, we propose to index the clusters' CBVs instead of the data points themselves. As a result, our index structure is not only simple, but also compact. The most convenient data structure that can efficiently accommodate such a small number of clusters is a sorted array, called array-index. The idea is to map the clusters' CBVs from the 2dimensional SOM grid into a 1-dimensional distance space, as discussed below.

3.3.1 Reference Point Selection. For the image database that we used in our experiments, we found that selecting a reference node $\mathbf{R}$ at the center of all nodes in the SOM grid (called, center $\mathbf{R}$ ) leads to a slightly faster search time than selecting a reference node at the edge of all nodes in the SOM grid (called, edge $\mathbf{R}$ ). That is because a center $\mathbf{R}$ leads to a more sparse mapping (less overlapping between clusters); thus, for a given query $\mathbf{q}$ the search space decreases. 
3.3.2 Distance Computation. After selecting $\mathbf{R}$, we compute the Euclidean distance $D\left(\mathbf{R}, \mathbf{m}_{\mathbf{i}}\right)$ between $\mathbf{m}_{\mathbf{i}}$ of each $\mathrm{CBV}$ and $\mathbf{R}$. By computing these distances, we actually map the CBVs into a one-dimensional distance space as illustrated in Figure 1. Such mapping is necessary for the following reasons:

(1.) Arranging the CBVs in a 1-dimensional distance space enables us to use the fast binary search algorithms to find a certain cluster in $O\left(\log N_{C}\right)$ time, where $N_{C}$ is the number of clusters.

(2.) During clustering, the SOM cannot position all similar clusters next to each other in the grid due to its geometry; therefore, mapping those clusters into a 1-dimensional distance space corrects clusters ' layout by bringing together similar clusters next to each other. However, clusters that are equidistant from $\mathbf{R}$, although not necessarily similar, are placed next to each other in the 1-dimensional space. These equidistant clusters are pruned during the $\mathrm{KNN}$ search as we will explain in Section 4.

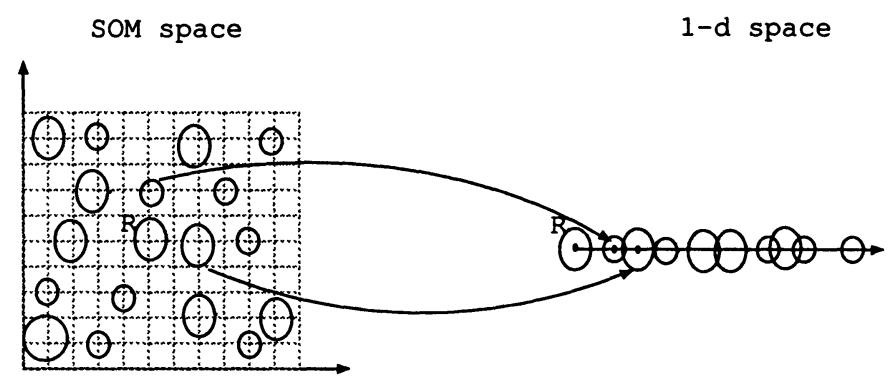

Figure 1. Mapping SOM clusters into a 1-dimensional distance space.

It is worth mentioning that a SOM can map clusters into a 1-dimensional space (instead of into a 2-dimensional space), but the one dimensional space will have the same problem of the 2-dimensional space where similar clusters can not be positioned next to each other. Thus, a remapping is necessary.

3.3.3 array-index Construction. The computed distances $D\left(\mathbf{R}, \mathbf{m}_{\mathbf{i}}\right)$ are inserted into the array-index. Along with each distance, a pointer to the best-matching-list (BML) of the corresponding cluster is set. The BML of cluster $C_{i}$ is a list of images associated the $C_{i}$. Also, for every $C_{i}$, we compute its radius $r_{i}$ that reflect the size of $C_{i}$ (see Section 4.1 for a formal definition of $r_{i}$ ). The constructed array-index is very small in size since it only contains three fields (distance, pointer, radius) for every generated cluster. Therefore, the array-index can be updated, resized or rebuilt with a very small overhead that is compromised by the fast search time it provides. 
Most KNN algorithms either require a storage space exponential in dimension, or require a query time not much better than a linear scan [8]. In our method, the size of the array-index does not depend on the dimensions of data, but it requires a storage space that is linear in the number of clusters, as opposed to dimensions.

\section{K-NEAREST NEIGHBORS SEARCH}

Given a query image $q$, we want to retrieve the exact $\mathrm{KNN}$ images. Our $\mathrm{KNN}$ search algorithm starts by finding the winner node (WN), which is a node with the most similar CBV to q, on the array-index. Then, as shown in Figure 2, the algorithm continues the search for similar clusters on both the left and right directions of the WN. During the search, dissimilar clusters are pruned based on some pruning condition (see Equation 4). Based on an ending condition (see Equation 5), the search ends when all $\mathrm{KNN}$ images are retrieved and further checking of new clusters does not result in new KNN answer images. Before, discussing the details of our KNN search algorithm, we introduce the pruning and ending conditions.

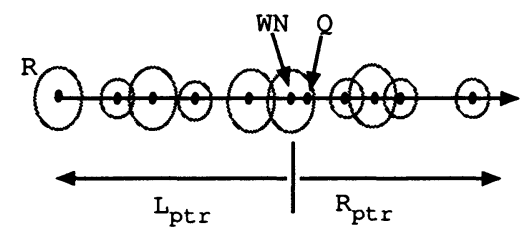

Figure 2. Searching to the left and right of the winner node (WN).

\subsection{Conditions for KNN Search}

To achieve efficient computation of the exact $\mathrm{KNN}$ answer points (i.e. images), our $\mathrm{KNN}$ search algorithm requires conditions on which pruning dissimilar clusters and ending the $\mathrm{KNN}$ search are based. Let $r_{i}$ be the radius of cluster $C_{i}$ and $r_{\max }$ be the largest radius among the radii of all clusters. Formally,

Definition 1 Let $P$ be a set of points in $C_{i}$, then, radius $r_{i}$ is the largest distance between the $C B V \mathbf{m}_{i}$ of $C_{i}$ and any point $p \in P$.

$$
r_{i}=\left\{D\left(\mathbf{m}_{i}, p\right) \mid p, p ́ p \in P: p \neq p ́ p\left(\mathbf{m}_{i}, p\right) \geq D\left(\mathbf{m}_{i}, \not{p}\right)\right\}
$$

Definition 2 Let $S$ be a set of radii of all clusters, then $r_{\text {max }}$ is the maximum radius in $S$. 


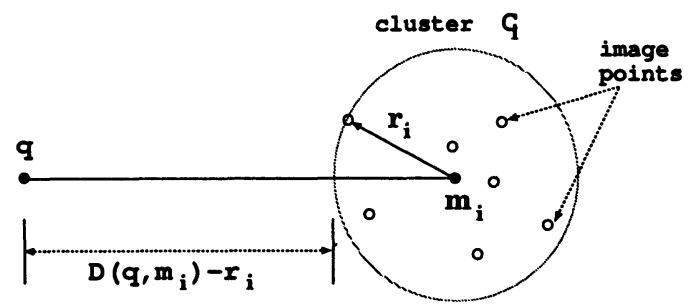

(a)

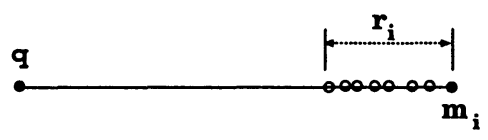

(b)

Figure 3. (a) An illustration of a radius of a cluster, (b) the image points on the 1-dimensional distance space.

$$
r_{\max }=\max \left\{r_{i} \mid r_{i} \in S\right\}
$$

Figure 3 shows the mapping of image points, which are associated with cluster $C_{i}$, onto the 1-dimensional distance space based on their Euclidean distances from $\mathbf{m}_{i}$. Clearly, image points that are equidistant from $\mathbf{m}_{i}$ are mapped to the same position on the 1-dimensional distance space.

4.1.1 Pruning Condition. Let $D_{k n n}^{c}$ be the current largest distance in the KNN list. Hence, cluster $C_{i}$ is pruned if the distance between $q$ and the closest point in $C_{i}$ to $\mathrm{q}$ is greater than $D_{k n n}^{c}$ (see Figure 3.a). Formally,

Condition 1 During the $K N N$ search, cluster $C_{i}$ is pruned if the following condition holds true:

$$
\left|D\left(\mathbf{q}, \mathbf{m}_{i}\right)\right|-r_{i}>D_{k n n}^{c}
$$

To assure that our pruning condition does not miss any qualifying clusters, we need to prove that cluster $C_{i}$ is pruned only if the distance $D_{k n n}^{c}$ is less than, or equal to, the distance between $\mathrm{q}$ and any point in $C_{i}$. Formally, we need to prove:

Lemma $1 D_{k n n}^{c} \leq D(\mathbf{q}, \mathbf{p})$, where $\mathbf{p} \in P$, and $P$ is a set of all image points in the pruned $C_{i}$.

Proof: By Definition $1, r_{i}$ is the largest distance $D\left(\mathbf{m}_{i}, \mathbf{p}\right)$, where $\mathbf{p} \in P$ and $P$ is a set of all points in the pruned $C_{i}$. Refer to Figure 3 , if $\mathbf{p}$ is on the circumference of $C_{i}$, then $\mathbf{p}$ is at distance $r_{i}$ from $\mathbf{m}_{\mathbf{i}}$; thus, $\left|D\left(\mathbf{q}, \mathbf{m}_{i}\right)\right|-$ $r_{i}=D(\mathbf{q}, \mathbf{p})$. Otherwise, if $\mathbf{p}$ is inside the perimeter of $C_{i}$, then $\mathbf{p}$ is at a distance less than $r_{i}$ from $\mathbf{m}_{\mathbf{i}}$; thus, $\left|D\left(\mathbf{q}, \mathbf{m}_{i}\right)\right|-r_{i}<D(\mathbf{q}, \mathbf{p})$. And, since 
$\left|D\left(\mathbf{q}, \mathbf{m}_{i}\right)\right|-r_{i}>D_{k n n}^{c}$ (see Equation 4), we conclude that $D_{k n n}^{c} \leq D(\mathbf{q}, \mathbf{p})$. Proof is Complete.

4.1.2 Ending Condition. Most of the previous KNN search algorithms, such as [14], traverses the whole index structure, except for the pruned branches; as a result, a large number of nodes are accessed. Therefore, we need to determine a condition on which the $\mathrm{KNN}$ search ends as soon as the exact $\mathrm{KNN}$ answer points are retrieved without the need to traverse the whole index structure.

To guarantee that no qualifying image points are missed, the search should not end if there exist a cluster overlapping $D_{k n n}^{c}$ because there might be an image point, in the overlapped portion of the cluster, whose distance from $q$ is smaller than $D_{k n n}^{c}$. That is we need to check all clusters whose $\mathbf{m}_{j}$ are in the vicinity of $D_{k n n}^{c}$ point up to $r_{\max }$ (that is $D_{k n n}^{c}+r_{\max }$ ) because $r_{\max }$ specifies the position of the farthest possible $\mathbf{m}_{j}$ of $C_{j}$ that might be overlapping with $D_{k n n}^{c}$. Formally, we state the ending condition of the KNN search as follows:

Condition 2 The KNN search ends when the following condition holds true in both the left and right directions:

$$
D_{k n n}^{c}+r_{\max }<\left|D\left(\mathbf{q}, \mathbf{m}_{i}\right)\right|
$$

Let the largest distance in the final $\mathrm{KNN}$ list be $D_{k n n}^{f}$. To guarantee that the $\mathrm{KNN}$ search stops without missing any qualifying image points, we need to prove that $D_{k n n}^{f}$ is less than, or equal to, the distance between $q$ and any image point in the set of all unchecked clusters. Formally, we need to prove that:

Lemma $2 D_{k n n}^{f} \leq D(\mathbf{q}, \mathbf{u})$, where, $\mathbf{u}$ is a point such that $\mathbf{u} \in U$ and $U$ is a set of all unchecked clusters.

Proof: By Definition 2, $r_{\max }$ is the maximum radius among the radii of all clusters. For any unchecked cluster that is overlapping with $D_{k n n}^{c}$, we find $r_{\max } \geq \max \left\{|| D\left(\mathbf{q}, \mathbf{m}_{i}\right)\left|-D_{k n n}^{c}\right|\right\}$, where $\mathbf{m}_{i}$ is the CBV of an unchecked cluster. That means, by checking all clusters whose $D\left(\mathbf{q}, \mathbf{m}_{i}\right)$ is less than, or equal to, $D_{k n n}^{c}+r_{\max }$ we can guarantees that no other cluster $C_{j} \in U$ has an image point $\mathbf{u}$ with $D(\mathbf{q}, \mathbf{u})$ less than $D_{k n n}^{c}$. Therefore, $D_{k n n}^{f}$ is less than, or equal to, the distance between $q$ and any image point $u$ in the set of unchecked clusters. Proof is complete.

\subsection{KNN Search of array-index}

Let's first explain some of the routines and notations in the KNNSSearch algorithm, which is shown below. The KNN_list keeps the distances of, and 
pointers to, the $K$ most similar images to q. The update_KNN_list $\left(C_{p t r}\right)$ routine computes the distances between $q$ and each of the image points associated with the cluster pointed at by $C_{p t r}$ and updates the $K N N$ _list according to the computed distances. Lptr and Rptr are pointers used to traverse the array-index toward the left and the right of WN, respectively.

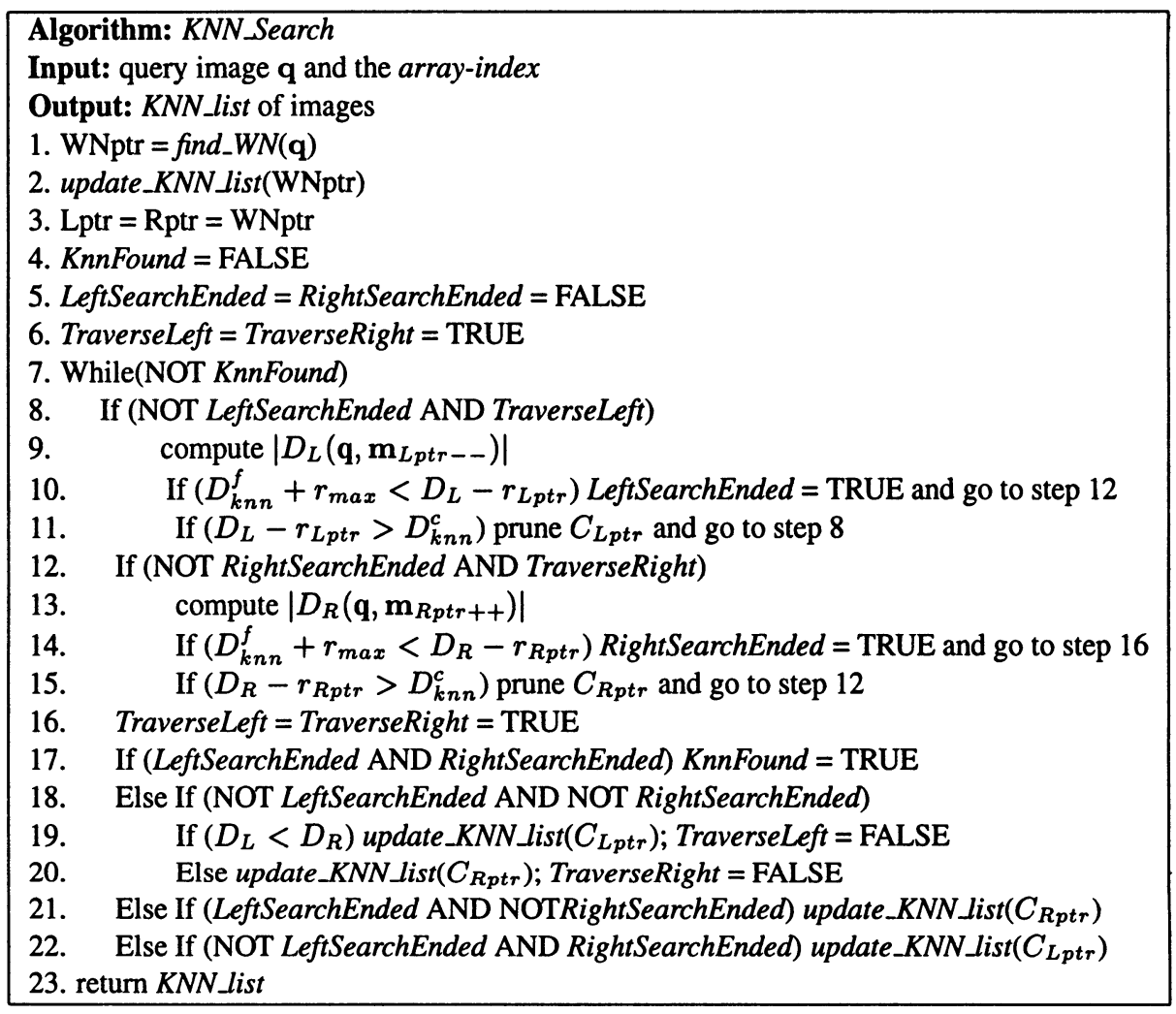

The first essential step in the KNNSSearch is to find WN, which is a cluster whose $\mathrm{CBV}$ is the most similar to q, as in line 1. In line 2, the $K N N \_$list is updated with the image points associated with the WN. Line 3 initializes Lptr and Rptr to the value of the pointer that points to WN. Lines 4-6 defines boolean variables used to control the ending of a search, pruning of dissimilar clusters, and traversal to the left and right of the WN. The search for KNN points is contained in the loop between lines 7-23. Lines 8-11 traverse the array-index towards the left of WN. The distance between $\mathbf{q}$ and the $\mathbf{m}_{\text {Lptr }}$ of the next unvisited cluster to the left of WN is computed, as shown in line 9. Then, in line 10, the ending condition for the left traversal is checked and the left traversal is ended if the condition holds true. Otherwise, if the left traversal is not ended, the currently visited cluster is checked against the pruning condition, if it holds true, the currently visited cluster is pruned and the algorithm advances 
to the next unvisited cluster in left direction. The right traversal (lines 12-15) is similar to that of the left traversal.

Lines 16-17 check if both traversal directions (left and right) have been ended. If so, the search ends and the KNN_Search algorithm returns the KNN_list. In lines 18-20, if the left traversal and right traversal have not been ended, then the KNN_list is updated with one of the two currently visited cluster (the one with the smaller distance from q), then the traversal in the opposite direction is blocked temporarily, so that the next iteration of the algorithm fetches the next cluster in the direction of the currently visited closer cluster (the one with the smaller distance) and keeps the currently visited farther cluster for the next comparison. Line 21 handles the case when the left traversal is ended and the search is advancing only in the right direction, thus, the KNN_Search algorithm updates the KNN_list with the next qualifying cluster on the right direction. Similarly, in lines 22 , the search only advances in the left direction. In line 23, the $K N N \_$list is returned with the exact $\mathrm{KNN}$ answer points.

\section{EXPERIMENTS}

We performed our experiments on collected images from publicly available image databases; $H^{2}$ Soft [18] and Stanford University [19]. The size of the collected image database is 40,000 artificial and natural images that span the following categories: landscapes, animals, buildings, people, plants, CG, etc. The image size is fixed at $128 \times 128$ pixels. We performed the experiments on an EPSON DIRECT PC (RAM: 128 Mbytes and CPU: Celeron $700 \mathrm{MHz}$ ) with a FreeBSD4.2 OS.

We extracted the color feature of each image using the Haar wavelets (see Section 3.1). The color space used in this paper is the YIQ color space since it exploits certain characteristics of the human eye, where $\mathrm{Y}$ carries luminance information and I and Q carry color information and some luminance information. The color feature for each image is represented by a $d$-dimensional vector. To demonstrate the efficiency of our $\mathrm{KNN}$ search algorithm, we generated 3 databases with different dimensionalities: 3,12 , and 48 dimensions by applying a 7-level, 5-level, and 3-level decomposition, respectively, of the Haar wavelet transform on the image data.

From each database $(3,12$, and 48 dimensions), we generated different test data sets $1 \mathrm{~K}, 5 \mathrm{~K}, 10 \mathrm{~K}, 20 \mathrm{k}, 30 \mathrm{k}, 40 \mathrm{k}$ vectors, where $\mathrm{k}=1000$. Each of these data sets was clustered by a SOM, as explained in Section 3.2. As shown in Table 1, we specified two map sizes (numbers of SOM nodes) for each data set: 'fixed map size' and 'adaptive map size'.

Fixed and Adaptive Map Sizes: The fixed and adaptive map sizes of each set reflect the maximum number of possible clusters and are used as parameters for the SOM. Hence, for each data set we generated two sets of clusters. 
Table 1. Map sizes ('fixed' and 'adaptive') of each data set.

\begin{tabular}{c|c|c}
\hline Data set & \multicolumn{2}{|c}{ Map size } \\
\cline { 2 - 3 } & fixed map size & adaptive map size \\
\hline 1000 & $20 \times 20$ & $30 \times 30$ \\
5000 & $30 \times 30$ & $70 \times 70$ \\
10000 & $30 \times 30$ & $100 \times 100$ \\
20000 & $30 \times 30$ & $130 \times 130$ \\
30000 & $30 \times 30$ & $170 \times 170$ \\
40000 & $30 \times 30$ & $200 \times 200$ \\
\hline
\end{tabular}

The number of nodes of the 'fixed map size' is small as compared to the cardinality of (number of images in) the database; thus, the number of generated clusters is almost equal to the specified number of nodes. On the other hand, for the 'adaptive map size', the number of specified nodes is equal to the number of images in the corresponding data set; thus, clustering the data generated empty nodes (nodes containing no images). As illustrated in Figure 4, the number of generated clusters in the 'adaptive map size' is adaptive to the size of the database (the number of clusters increases as the size of the database increases), where the number of generated clusters is decided by the SOM algorithm based on the features of input database. Also, we notice that the 12-dimensional data sets have lower number of empty nodes than those of the 3-dimensional and 48-dimensional data sets. That is because the data sets in the 12-dimensional space are more sparse, hence occupies more clusters. On the other hand, the data sets in 3-dimensional space suffers from high degree of detail loss leading to large number of false similaritiss and bigger, but fewer, clusters. Also, in the 48-dimensional space, there is more overlap between image vectors due to the 'dimensionality curse' leading to less number of clusters as compared to the 12-dimensional clusters.

Search Efficiency: To analyze the effect of dimensionality on the KNN search time, we measured the search time of data sets of different sizes and different dimensionalities, as shown in Figure 5. First, we randomly selected 10 images from the database and issued a query to retrieve the $\mathrm{KNN}$ (where, $\mathrm{K}=1$ and $\mathrm{K}=100$ ) answer images for each of the different sizes and different dimensionalities. The search times shown in Figure 5.a and 5.b are the average of 10 queries. We notice that for both $\mathrm{K}=1$ and $\mathrm{K}=100$ the 12-dimensional data sets have lower $\mathrm{KNN}$ search time than the 3-dimensional and 48-dimensional data sets especially when the size of the data sets increases $(\geq 1000)$. That is because the 12-dimensional data is more sparse, thus occupying more clusters; as a consequence, when a query is issued only a fewer number of clusters is needed to be accessed to retrieve the KNN images as compared to the other dimensionalities. 


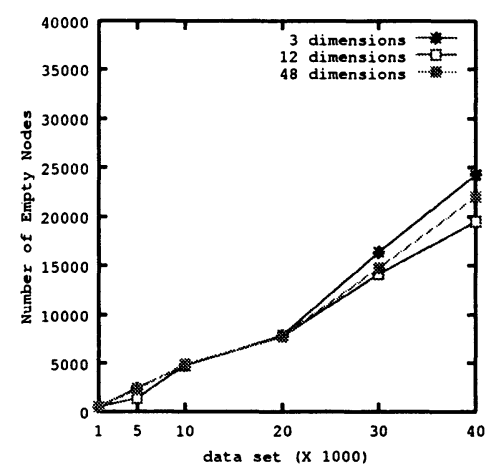

Figure 4. The number of empty nodes in the three databases: 3-dim DB, 12-dim DB, and 48-dim DB.

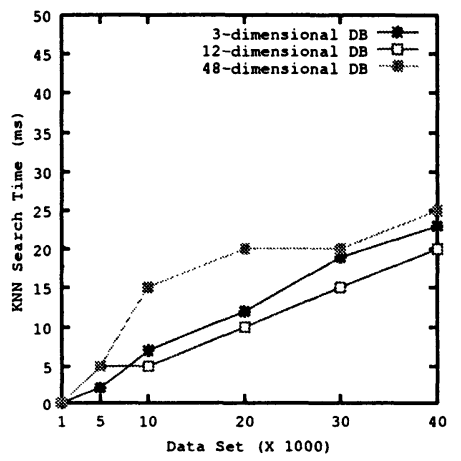

(a)

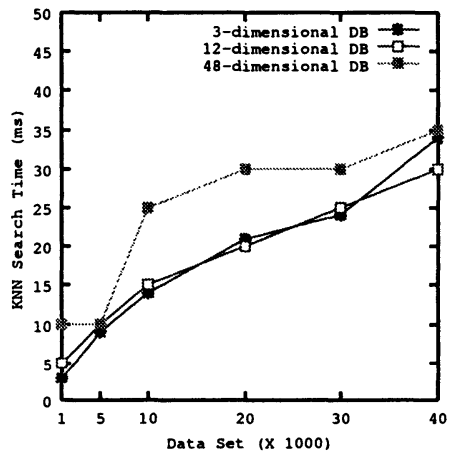

(b)

Figure 5. Average $\mathrm{KNN}$ search time versus database size when (a) $\mathrm{K}=1$, and (b) $\mathrm{K}=100$.

To prove the above reasoning, we measured the percentage of visited nonempty (associated with one or more images) clusters accessed to retrieve the $\mathrm{KNN}$ images to a given query (see Figure 6). We notice that, for both cases $\mathrm{K}=1$ and $\mathrm{K}=100$, the percentage of visited nodes of the 12-dimensional data is lower than those of the 3-dimensional and 48 dimensional data especially for data sets $\geq 10000$ images.

To analyze the dynamics of the array-index method, first, we clustered a small data set, which is 5000 image, using the 'fixed map size' $(30 \times 30)$ as a parameter for the SOM algorithm and measured the KNN search time. Then, as shown in Figure 7, we increased the size of the database to 10000, 20000, 30000 , and 40000 images and measured the KNN search time at each database size. The test shown in Figure 7 is for a database with 48 dimensions and $K=1$. We notice, for the 'fixed map size', as the database size increases the KNN 


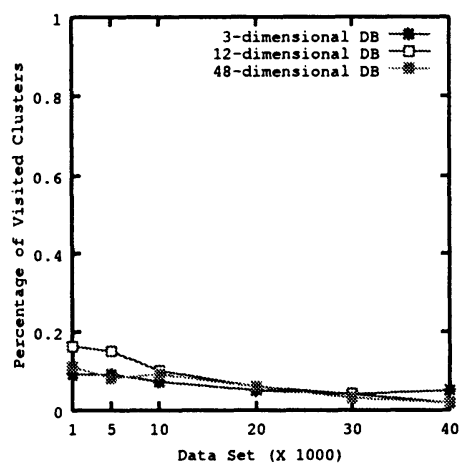

(a)

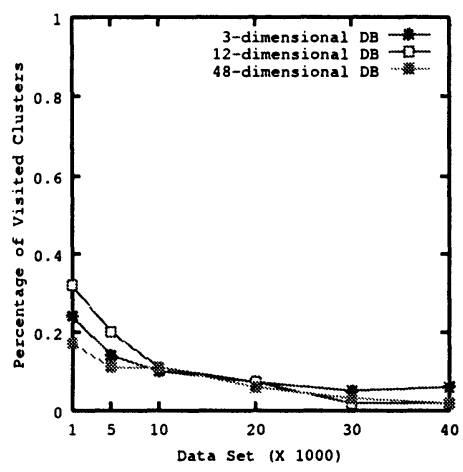

(b)

Figure 6. The percentage of the accessed database (visited nodes) versus database size when (a) $\mathrm{K}=1$, and (b) $\mathrm{K}=100$.

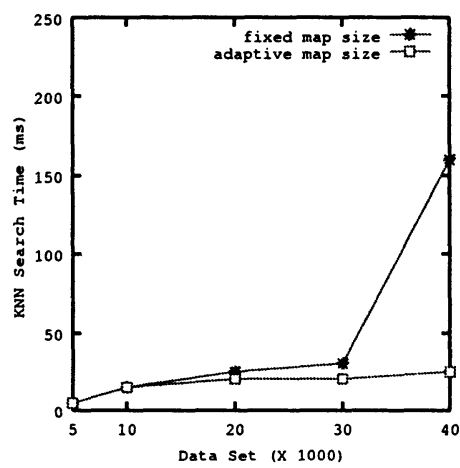

Figure 7. Comparison between the 'fixed map size' and 'adaptive map size" to show the dynamic extent of the array-index method (dimensions $=48$, and $K=1$ ).

search time increase slightly up to the database size of 30000 , and beyond that size the KNN search time shoots up with great increases. The reason is that beyond the database size of 30000 images the size of clusters become very big (containing large number of images); thus, the number of false alarms for a given query increase greatly in the clusters leading to many distance computations to remove those false alarms and hence increases the KNN search time. Thus, we conclude that beyond the database size of 30000 the system should recluster the database with an increase in the map size (number of clusters). As a comparison, in Figure 7, we show the KNN search time of the same data sets but with different number of clusters (using the 'adaptive map size', see Table 1). 


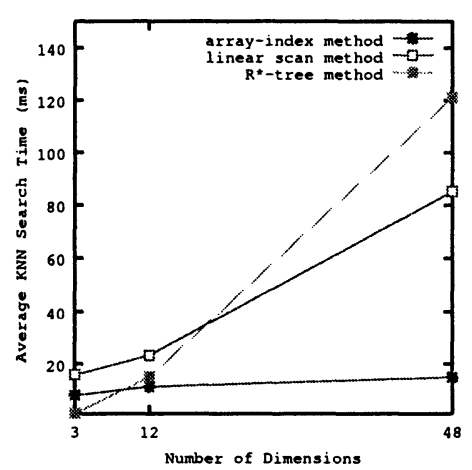

(a)

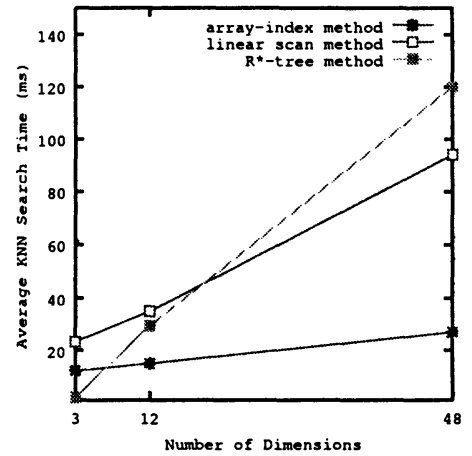

(b)

Figure 8. Comparison between the array-index method and a linear scan method in terms of $\mathrm{KNN}$ search time versus number of dimensions: (a) for $\mathrm{K}=1$, and (b) for $\mathrm{K}=100$.

Comparative Study: According to [8], most KNN search algorithms, require a search time not much better than a linear scan. We compared the array-index method with other well known methods $\left(\mathrm{R}^{*}\right.$-tree and linear scanning), which serve as a stick-yard to evaluate our method, in terms of $\mathrm{KNN}$ search time. Figure 8 shows the comparison between the three methods for $\mathrm{K}=1$ and $\mathrm{K}=100$ using a database of size 20000 images. Obviously, the arrayindex method outperforms the other two methods, $\mathrm{R}^{*}$-tree and linear scanning, particularly, at high-dimensionalities ( $\geq 12$ dimensions $)$, which are the bottlenecks for most KNN search algorithms. The KNN search time of the linear scanning methods increases linearly with the number of dimensions and the search time of the $\mathrm{R}^{*}$-tree increases exponentially with the number of dimensions. On the other hand, the array-index method shows more robustness to the number of dimensions since the KNN search time only increases slightly as the dimensionality increases. Clearly, the array-index method reduces the search space greatly due to its simple and compact structure.

\section{CONCLUSION}

We presented a simple and efficient method, called array-index, to retrieve exact $K$-nearest neighbors for a given query. The array-index method is based on clustering the data and then indexing the clusters' representative vectors instead of the data points themselves; thus, reducing the size of the index structure. Although, we clustered the database using a SOM algorithm, the array-index method will works well with any clustering technique. The compactness and the ordering of clusters in the array-index are the main reasons behind the fast KNN search time as compared to other known methods. The compactness of the array-index enabled us to put the whole index structure 
in memory leading to an elimination of $\mathrm{I} / \mathrm{O}$ cost, and the ordering of clusters based on their distances from a reference point helped reducing the percentage of accessed database necessary to determine the exact KNN answer images.

\section{REFERENCES}

[1] P.Korn, N.Sidiropoulos, C.Faloutsos, E.Siegel, Z.Protopapas. Fast and Effective Retrieval of Medical Tumor Shapes. IEEE Trans. on Knowledge and Data Engineering, Vol.10, No.6. Nov/Dec 1998.

[2] E.Keogh, K.Chakrabarti, S.Mehrotra, M.Pazzani. Locally Adaptive Dimensionality Reduction for Indexing Large Time Series Databases. ACM SIGMOD, 2001.

[3] C.Faloutsos and K.Lin. A Fast Algorithm for Indexing, Data-Mining and Visualization of Traditional and Multimedia Datasets. ACM SIGMOD, May 1995.

[4] N.Beckmann, H.Kriegel, R.Schneider, B.Seeger. $R^{*}$-tree: An Efficient and Robust Access Method for Points and Rectangles. ACM SIGMOD, pp. 322-331, May 1990.

[5] S.Berchtold, D.Keim, H.P.Kriegel. The X-tree: An Index Structure for High-Dimensional Data. VLDB 1996.

[6] S.Berchtold, C.Bohm, D.Keim, H.P.Kriegel. A Cost Model for Nearest Neighbor Search in HighDimensional Data Spaces. ACM SIGACT-SIGMOD-SIGART, 1997.

[7] S.Berchtold, B.Ertl, D.Keim, H.P.Kriegel, T.Seidl. Fast Nearest Neighbor Search in HighDimensional Space. Int'l conf. on Data Eng. (ICDE), 1998.

[8] J.M.Kleinberg. Two Algorithms for Nearest Neighbor Search in High Dimensions. 29th ACM Symposium on Theory of Computing, 1997.

[9] C.Faloutsos. Searching Multimedia Databases By Content. Kluwer Academic Publishers, Boston, USA, 1996.

[10] Y.Sakurai, M.Yoshikawa, S.Uemura. The A-tree: An Index Structure for High-Dimensional Spaces Using Relative Approximation. VLDB 2000.

[11] M. Flickner, H. Sawhney, W. Niblack, J. Ashley, Q. Huang, B. Dom, M. Gorkani, J. Hafner, D, Lee, D. Perkovic, D. Steele, P. Yanker. Query by Image and Video Content: The QBIC System. IEEE, Sept. 1995.

[12] K.Chakrabarti, S.Mehrotra. Local Dimensionality Reduction: A New Approach to Indexing High Dimensional Space. 26th VLDB, Egypt, 2000.

[13] C.Faloutsos, M.Ranganathan, Yannis Manolopoulos. Fast Subsequence Matching in Time-Series Databases. ACM SIGMOD, 1994.

[14] N.Roussopoulos, S.Kelley, F.Vincent. Nearest Neighbor Queries. ACM SIGMOD, 1995.

[15] S.Brin. Near Neighbor Search in Large Metric Spaces. VLDB 1995.

[16] A.Natsev, R.Rastogi, K.Shim. WALRUS: A Similarity Retrieval Algorithm for Image Databases. SIGMOD record, vol.28, no.2, pp.395-406, Philadelphia, PA, 1999.

[17] T.Kohonen. Self-Organizing Maps. Springer-Verlag, 1997. 2nd extended edition.

[18] $H^{2}$ soft, http://www.h2soft.co.jp.

[19] Stanford University, http://www-DB.Stanford.EDU/IMAGE/. 